\title{
Synthesis and characterization of partially coherent beams with propagation-invariant transverse polarization pattern
}

\author{
Victoria Ramírez-Sánchez ${ }^{a}$, Gemma Piquero ${ }^{\mathrm{a}, *}$, Massimo Santarsiero ${ }^{\mathrm{b}}$ \\ a Departamento de Óptica, Facultad de Ciencias Físicas, Universidad Complutense de Madrid, 28040 Madrid, Spain \\ b Dipartimento di Fisica, Università Roma Tre, and CNISM, Via della Vasca Navale 84, 00146 Rome, Italy
}

\section{A R T I C L E I N F O}

Article history:

Received 10 July 2009

Received in revised form 10 December 2009

Accepted 21 April 2010

\begin{abstract}
A B S T R A C T
Partially coherent beams, whose transverse polarization pattern remains invariant upon paraxial propagation, are synthesized and characterized. Synthesis is performed by imposing a spiral-like polarization profile to a rotationally symmetric partially coherent light source. Irradiance and polarization profiles of the propagated beam are detected at different transverse planes, both in the near and in the far zone, and are compared to the theoretical ones. Furthermore, overall parameters, measuring the circular, radial and azimuthal polarization contents across the beam profile, are used to characterize the generated beam from a global point of view.
\end{abstract}

(c) 2010 Elsevier B.V. All rights reserved.

\section{Introduction}

There is an increasing interest in beams with a non-uniform polarization distribution across the plane orthogonal to the mean propagation direction, and much research is devoted to the study of changes that occur when a partially and/or non-uniformly polarized beam propagates in free space or through optical systems (see for example Refs. [1-15]). In particular, it is well known that a nonuniformly polarized beam generally changes its polarization pattern upon propagation, even in the case of perfectly polarized and deterministic field distributions in paraxial conditions. Of course, this happens because, in general, the two orthogonal transverse components of the electric field change in different ways upon propagation, so that the polarization pattern of the propagated field does not reproduce the initial one. Significant exceptions are radially or azimuthally polarized beams, or their generalization, the so-called spirally polarized beams (SPB) [5,16-18], whose electric field lines across a transverse plane are logarithmic spirals, and that include, as limiting cases, both azimuthally and radially polarized beams. Beams of these types have been also shown to be useful in applications, such as microscopy, material processing, manipulation of atoms or molecules (see Ref. [14] and references therein).

On the other hand, when non-deterministic fields are considered, a richer behaviour is observed in their polarization and coherence features during propagation. One of the main tasks in this subject is to derive the conditions that a general field must satisfy in order to maintain invariant its polarization characteristics when it freely propagates [6,10-13]. In particular, Tervo [6] derived the conditions

\footnotetext{
* Corresponding author. Tel.: +34 91 39445454; fax: +34 913944683. E-mail address: piquero@fis.ucm.es (G. Piquero).
}

under which an electromagnetic partially coherent source gives rise to an azimuthally polarized field at any transverse plane orthogonal to the mean propagation direction. Such polarization-invariance conditions can be extended [12] to the whole class of partially coherent spirally polarized beams.

Most of the above studies are theoretical and, to our knowledge, there are no papers yet in the literature reporting experimental synthesis of partially coherent beams with propagation-invariant polarization patterns.

The aim of this work is to present an experimental procedure for the synthesis of partially coherent beams of this type. In particular, a beam endowed with a spiral-like polarization transverse profile has been synthetized and characterized. Characterization has been performed at different transverse planes both by detecting the local state of polarization point by point across the beam profile and, from a global point of view, by means of some recently introduced overall parameters $[19,20]$.

The paper is arranged as follows. In Section 2, we recall the polarization-invariance conditions that the source must satisfy in order to produce a partially coherent beam with spiral-like polarization distribution across any transverse plane upon free propagation. A general procedure for synthesizing such beams is given in Section 3. In Section 4, experimental results are presented, concerning the transverse polarization pattern and irradiances of the propagated beam, as well as the values of the above overall parameters, and are compared to the theoretical ones. The main conclusions are reported in Section 5.

\section{Preliminaries}

Let us begin with a paraxial quasi-monochromatic partially coherent electromagnetic beam, described at the plane $z=0$ (which 
will be referred to as the source plane) by means of its cross-spectral density (CSD) matrix [21], that is

$\hat{W}\left(\boldsymbol{\rho}_{1}, \boldsymbol{\rho}_{2}, 0\right)=\left(\begin{array}{cc}\left\langle E_{x}\left(\boldsymbol{\rho}_{1}, 0\right) E_{x}^{*}\left(\boldsymbol{\rho}_{2}, 0\right)\right. & \left.E_{x}\left(\boldsymbol{\rho}_{1}, 0\right) E_{y}^{*}\left(\boldsymbol{\rho}_{2}, 0\right)\right\rangle \\ \left\langle E_{y}\left(\boldsymbol{\rho}_{1}, 0\right) E_{x}^{*}\left(\boldsymbol{\rho}_{2}, 0\right)\right. & \left.E_{y}\left(\boldsymbol{\rho}_{1}, 0\right) E_{y}^{*}\left(\boldsymbol{\rho}_{2}, 0\right)\right\rangle\end{array}\right)$

where $\rho=(\rho, \varphi)$ is the position vector across the transverse plane, $E_{x}$ and $E_{y}$ are the two transverse components of the electric field, the brackets denote ensemble averages, and the dependence on the temporal frequency has been omitted for brevity.

In Ref. [6], Tervo derived a necessary and sufficient condition for a beam to be azimuthally polarized at any propagation distance. He showed that, in such a case, the CSD matrix at $z=0$ must be of the form

$\hat{W}\left(\boldsymbol{\rho}_{1}, \boldsymbol{\rho}_{2}, 0\right)=W^{\mathrm{s}}\left(\boldsymbol{\rho}_{1}, \boldsymbol{\rho}_{2}, 0\right) \hat{P}\left(\varphi_{1}, \varphi_{2}\right)$,

with $W^{\mathrm{s}}\left(\boldsymbol{\rho}_{1}, \boldsymbol{\rho}_{2}, 0\right)$ being any scalar CSD function, depending only on the radial coordinates $\rho_{1}$ and $\rho_{2}$, and $\hat{\mathrm{P}}\left(\varphi_{1}, \varphi_{2}\right)$ is defined as

$\hat{P}\left(\varphi_{1}, \varphi_{2}\right)=\Phi\left(\varphi_{1}\right) \Phi^{\dagger}\left(\varphi_{2}\right)$

where $\Phi(\varphi)$ is the Jones vector corresponding to an azimuthally polarized field, i.e.,

$\Phi(\varphi)=\left(\begin{array}{c}-\sin \varphi \\ \cos \varphi\end{array}\right)$,

and the dagger denotes the adjoint. Eq. (2) states that any partially coherent field with propagation-invariant azimuthal polarization pattern must present perfect correlation between points belonging to circles $\rho=$ constant.

Condition in Eq. (2) can be generalized to the case of beams that remain spirally polarized upon free propagation [12]. In such a case, the matrix $\hat{P}$ that appears in Eq. (2) turns out to be

$\hat{P}\left(\varphi_{1}+\alpha, \varphi_{2}+\alpha\right)=\Phi\left(\varphi_{1}+\alpha\right) \Phi^{\dagger}\left(\varphi_{2}+\alpha\right)$,

where the angle $\alpha$ is related to the growth parameter of the polarization spirals. The polarization pattern described by the Jones vector appearing in Eq. (5) is sketched in Fig. 1. In particular, it can be seen, that for $\alpha=n \pi$, with $n=0,1,2 \ldots$, the beam is azimuthally

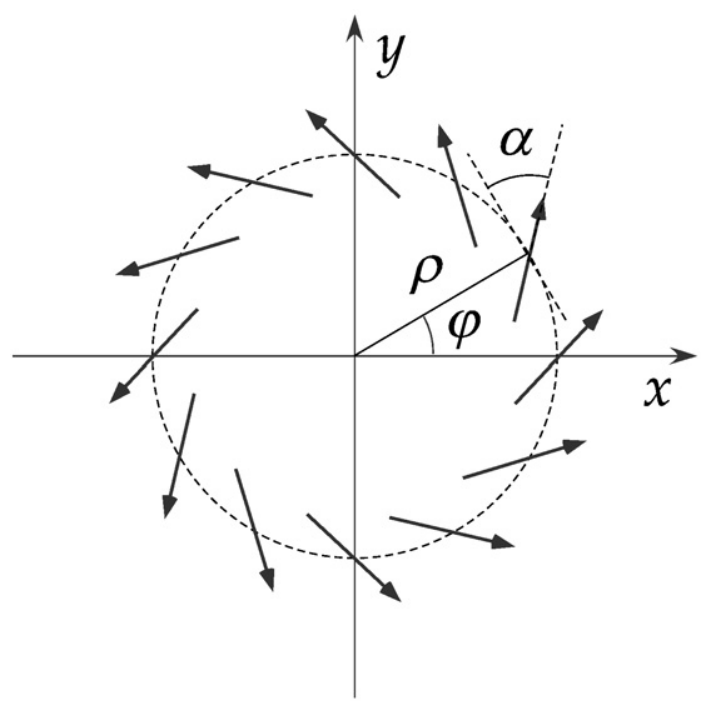

Fig. 1. Polarization pattern of a spirally polarized beam. polarized while for $\alpha=(2 n+1) \pi / 2$ the beam is radially polarized. The polarization-invariance condition then reads

$\hat{W}\left(\rho_{1}, \rho_{2}, 0\right)=W^{s}\left(\rho_{1}, \rho_{2}, 0\right) \hat{P}\left(\varphi_{1}+\alpha, \varphi_{2}+\alpha\right)$.

Let now this beam propagate at a certain distance $z$, in paraxial conditions. On using the Fresnel integral, and denoting by $\boldsymbol{r}=(r, \theta)$ the position vector across the plane $z=$ constant, the propagated CSD matrix turns out to be

$\hat{W}\left(\mathbf{r}_{1}, \mathbf{r}_{2}, z\right)=W^{\mathrm{s}}\left(r_{1}, r_{2}, z\right) \hat{P}\left(\theta_{1}+\alpha, \theta_{2}+\alpha\right)$,

with

$$
\begin{aligned}
& W^{\mathrm{s}}\left(r_{1}, r_{2}, z\right)=\left(\frac{k}{z}\right)^{2} \exp \left[\mathrm{i} \frac{k}{2 z}\left(r_{1}^{2}-r_{2}^{2}\right)\right] \\
& \quad \times \iint W^{\mathrm{s}}\left(\boldsymbol{\rho}_{1}, \boldsymbol{\rho}_{2}, 0\right) \exp \left[i \frac{k}{2 z}\left(\boldsymbol{\rho}_{1}^{2}-\boldsymbol{\rho}_{2}^{2}\right)\right] J_{1}\left(\frac{k}{z} r_{1} \boldsymbol{\rho}_{1}\right) J_{1}\left(\frac{k}{z} r_{2} \boldsymbol{\rho}_{2}\right) \boldsymbol{\rho}_{1} \boldsymbol{\rho}_{2} \mathrm{~d} \boldsymbol{\rho}_{1} \mathrm{~d} \boldsymbol{\rho}_{2},
\end{aligned}
$$

where $k$ is the wave number, $J_{1}$ is the Bessel function of the first kind and order 1 [22], and the integrals are extended over the whole source plane. As can be seen from Eq. (7), the polarization pattern remains invariant upon free propagation, regardless the value of $\alpha$.

\section{Synthesis}

According to the condition in Eq. (6), the scalar part of the CSD matrix of a beam, whose transverse polarization pattern remains of the spiral type upon propagation, must depend on the radial coordinates $\rho_{1}$ and $\rho_{2}$ only. This means that all points belonging to a circle $\rho=$ constant has to be perfectly correlated with one another. Such a requirement prevents the use of the van Cittert-Zernike theorem for the synthesis of such beams, as it is customary for all sources of the Schell-model type $[3,23,24]$, and a different approach must be used.

The synthesis can be realized, for instance, by superimposing a number, say $N$, of mutually uncorrelated perfectly coherent fields, all of them endowed with rotational symmetry and spiral polarization pattern. We can write such fields in the form

$\mathbf{U}_{n}(\boldsymbol{\rho})=\sqrt{\lambda_{n}} f_{n}(\boldsymbol{\rho}) \Phi(\varphi+\alpha)$,

where $n=1, \ldots, N$, the functions $f_{n}(\boldsymbol{\rho})$ are supposed to be normalized, and the quantity $\lambda_{n}$ gives account of the power content of the $n$th field. Furthermore, since the polarization vector is not defined at $\rho=0$, the condition $f_{n}(0)=0$ must be fulfilled.

Since the fields $\mathbf{U}_{n}$ are supposed to be mutually uncorrelated, the CSD matrix obtained from their superposition is of the form in Eq. (6), with

$W^{\mathrm{s}}\left(\boldsymbol{\rho}_{1}, \boldsymbol{\rho}_{2}, 0\right)=\sum \lambda_{n} f_{n}\left(\boldsymbol{\rho}_{1}\right) f_{n}^{*}\left(\boldsymbol{\rho}_{2}\right)$

This expression reduces to a Mercer-like expansion when the fields $f_{n}(\boldsymbol{\rho})$ coincide with the modes and $\lambda_{n}$ with the eigenvalues of $W^{\mathrm{s}}\left(\boldsymbol{\rho}_{1}, \boldsymbol{\rho}_{2}, 0\right)[25,26]$. In this sense, any scalar CSD depending only on the radial coordinates $\rho_{1}$ and $\rho_{2}$ can be synthesized following such an approach, and any spirally polarized partially coherent beam can be generated. It should be noticed, however, that neither the orthogonality among the fields, neither their mutual uncorrelation are necessary in the synthesis procedure.

In our experiment, two spirally polarized fields with rotationally symmetric amplitudes are superimposed by using the set-up sketched in Fig. 2. Two distinct He-Ne laser beams, linearly polarized along the vertical axis, are expanded, spatially filtered, and collimated by means of a microscope objective and a converging lens $\left(O_{i}\right.$ and $L_{i}$, respectively, with $i=1,2)$. They pass through two different annular 


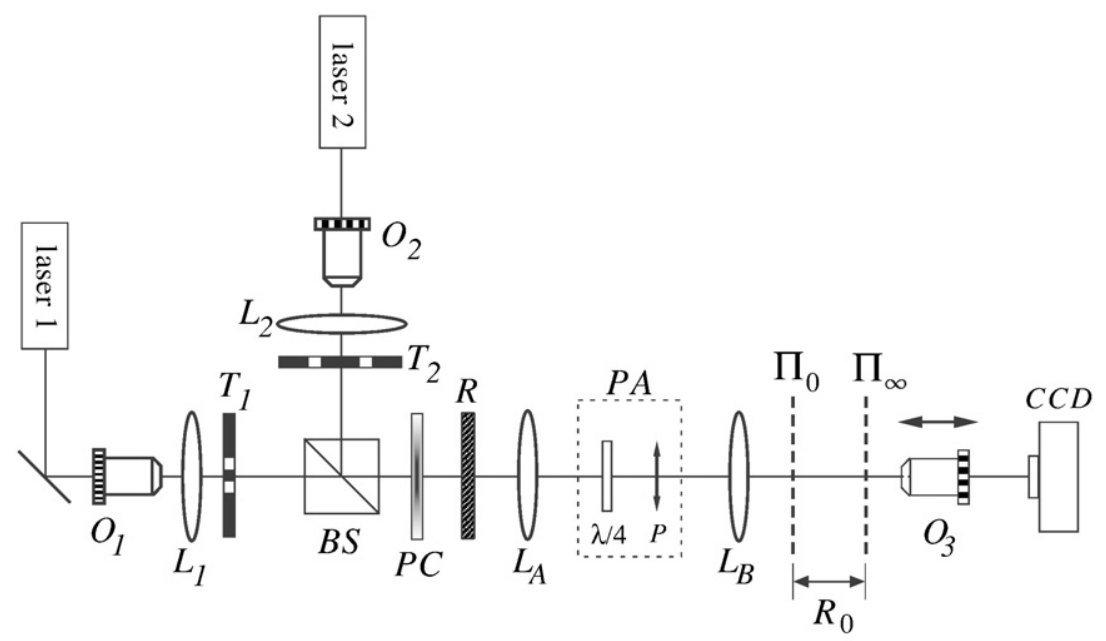

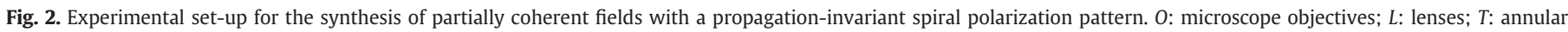
transmittances; $B S$ : beam splitter; PC: polarization converter; $R$ : polarization rotator; $P A$ : polarization analizer; $\lambda / 4$ : quarter-wave phase plate; and $P$ : linear polarizer.

apertures $T_{i}$ (Fig. 3), such that the output field distributions have analytical forms given by

$f_{i}(\mathbf{\rho})=A_{i}\left[\operatorname{circ}\left(\mathbf{\rho} / \mathbf{B}_{\mathbf{i}}\right)-\operatorname{circ}\left(\mathbf{\rho} / \mathbf{C}_{\mathbf{i}}\right)\right] ; \quad(i=1,2)$,

with $\operatorname{circ}(\rho / a)$ representing a clear circular aperture of radius $a$. In particular, the values $A_{1}=A_{2}, B_{1}=2.35 \mathrm{~mm}, C_{1}=1.50 \mathrm{~mm}$ and $B_{2}=3.20 \mathrm{~mm}, C_{2}=2.46 \mathrm{~mm}$ have been used.

The two beams are then superimposed by using a beam splitter (BS) and the images of $T_{1}$ and $T_{2}$ are formed onto the plane $\Pi_{0}$ by means of an optical system, consisting in the two converging lenses $L_{A}$ and $L_{B}$, with magnification $M=-0.30$. The plane $\Pi_{0}$ represents the source plane $(z=0)$ of the synthesized beam. Note that $f_{1}(\boldsymbol{\rho})$ and $f_{2}(\boldsymbol{\rho})$ in Eq. (11) represent non-overlapping annular distributions across the source plane, so that the fields at two points are perfectly correlated if the points belong to the same annulus, but they are completely uncorrelated if the points belong to different annulus. In particular, points along a circle are always perfectly correlated with one another.

In order for the source to be endowed with a spiral-like polarization pattern, we use first an Arcoptix liquid-crystal polarization converter $(P C)$, which locally rotates the incident linear polarization [27]. The Jones matrix describing the polarization converter can be written as

$\hat{A}=\left(\begin{array}{cc}\cos \varphi & -\sin \varphi \\ \sin \varphi & \cos \varphi\end{array}\right)$,

so that the field at the output of the PC turns out to be azimuthally polarized. After the $P C$, a polarization rotator $(R)$, consisting in two

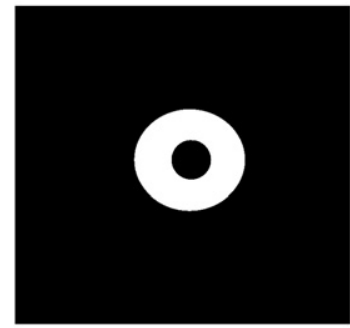

$T_{1}$

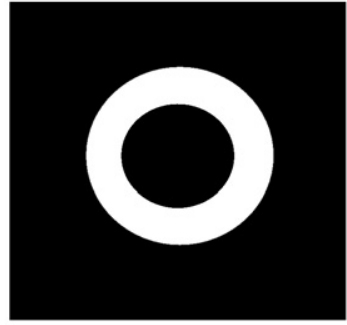

$T_{2}$
Fig. 3. Scheme of the transmittances used in the experiment. half-wave plates, whose fast axes form the angle $\gamma$ with one another (see Fig. 4), is placed [28]. The Jones matrix of this element is [28]

$\hat{R}=\left(\begin{array}{cc}\cos 2 \gamma & -\sin 2 \gamma \\ \sin 2 \gamma & \cos 2 \gamma\end{array}\right)$

and therefore it represents a local rotation by $2 \gamma$ of the incident polarization. In conclusion, at the plane $\Pi_{0}$ a partially coherent source with spiral polarization pattern, with $\alpha=2 \gamma$, is obtained. In our experiment, the angle between the two fast axes of the half-wave plates in the rotator has been set in such a way that $\alpha=-35^{\circ}$.

The back focal plane of the above optical system (i.e., the lenses $L_{A}$ and $L_{B}$ ) is $\Pi_{\infty}$, at a distance $R_{0}=240 \mathrm{~mm}$ from $\Pi_{0}$ (see Fig. 2). This means that both the annular fields imaged onto $\Pi_{0}$ present, at $z=0$, a negative curvature factor of the form $\exp \left[-\mathrm{i} k \rho^{2} / 2 R_{0}\right]$. Therefore, all propagation distances that would have been considered if the curvature was not present (between zero and infinity) are mapped onto a finite interval (between $\Pi_{0}$ and $\Pi_{\infty}$ ). On the other hand, the presence of a curvature factor in the propagated field does not affect its polarization characteristics.

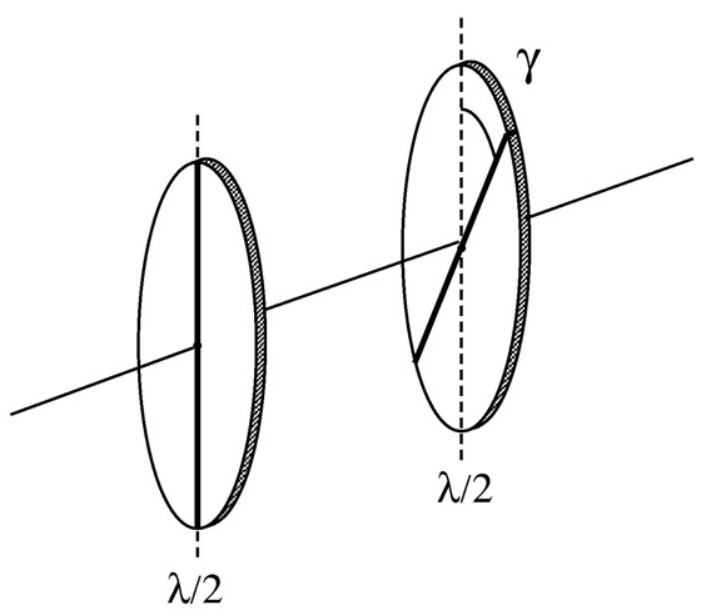

Fig. 4. The polarization rotator, consisting in two half-wave phase plates, $\lambda / 2$, whose fast axes form the angle $\gamma$ with one another. 
These concepts are quantified by the (generalized) Fresnel number [29], which in the present case can be defined as

$N_{F}=\frac{\left(M B_{2}\right)^{2}}{\lambda}\left(\frac{1}{z}-\frac{1}{R_{0}}\right)$,

which controls the shape of the transverse intensity profile. In particular, when $z=R_{0}$ we have $N_{F}=0$, meaning that $\Pi_{\infty}$ is the plane where the far-field conditions for the evaluation of the field radiated by the source are exactly met. Moving the observation plane toward the source leads to increasing the value of $N_{F}$, up to infinity (when $z=0)$.

The propagated intensity profiles are observed by means of the microscope objective $\mathrm{O}_{3}$, which images them onto the CCD sensor of a camera (Pulnix TM-765). $\mathrm{O}_{3}$ can be moved along the optical axis, so that profiles at different propagation distances can be acquired. Furthermore, a polarization analyzer $(P A)$, consisting in a quarter-wave plate $(\lambda / 4)$ and a linear polarizer $(P)$, is used for the measurement of the local Stokes parameters of the field across its transverse sections.

Results about the characterization of the generated beam will be presented in the following section.

\section{Characterization}

To experimentally study the characteristics of the synthesized beam upon propagation, the polarization distribution and some significant global parameters of the field across different transverse planes have been analyzed. All of them are calculated from the local Stokes parameters, $s_{i}(i=0,1,2,3)$ which, in turn, can be determined with a standard procedure on measuring the irradiance $I(r, \theta)$ for different choices of the parameters of the polarization analyzer (i.e., the orientation of the polarizer and the presence of the wave plate). With evident meaning of the symbols, we have [30]

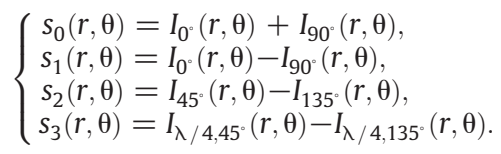

To give a first description of the polarization distribution of the synthesized beam across its transverse sections, we used Eq. (15) to evaluate the irradiance and the local polarization state at four different transverse planes: $z=0$ (i.e., the source plane) (a), two intermediate planes, $z=z_{1}=86 \mathrm{~mm}\left(N_{F} \simeq 11\right)$ (b) and $z=z_{2}=106 \mathrm{~mm}\left(N_{F} \simeq 7.7\right)$ (c), respectively, and the far-field plane $z=R_{0}=240 \mathrm{~mm}\left(N_{F}=0\right)$ (d). Results shown in Fig. 5 confirm that the spiral-like distribution of polarization remains almost invariant upon paraxial propagation. The small differences between experimental and expected patterns may be due to imperfections of the polarization converter $(P C)$ or to slight nonuniformities in the irradiance profile of the input beam. To compare the above plots with the theoretical ones, the latter have been reported in Fig. 6. As can be seen, the agreement between theory and experiment is quite good. a

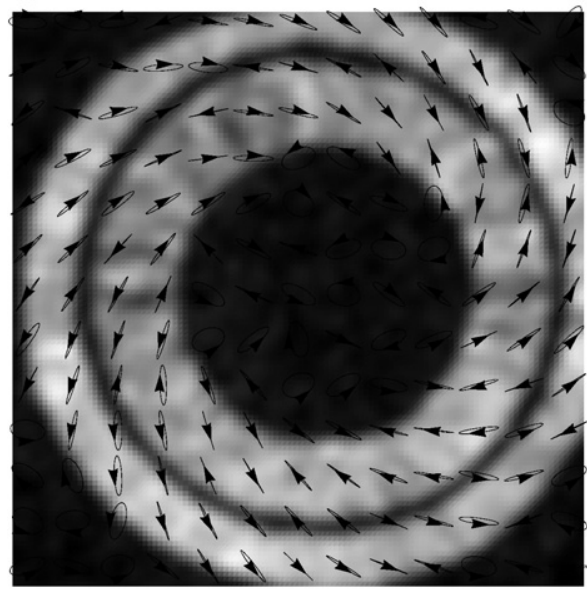

C

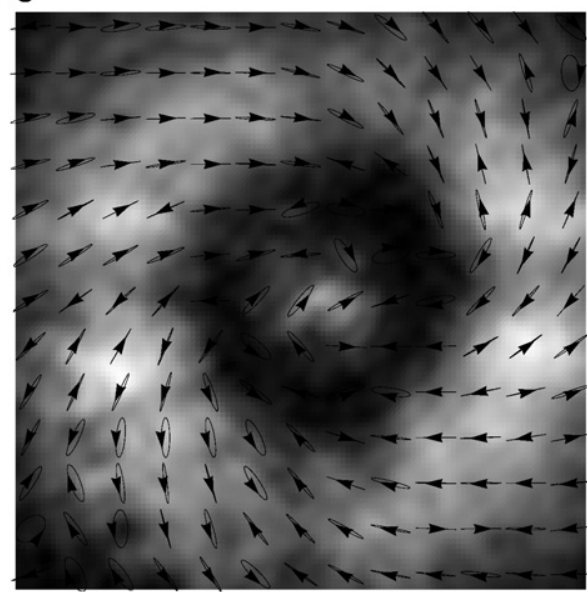

b

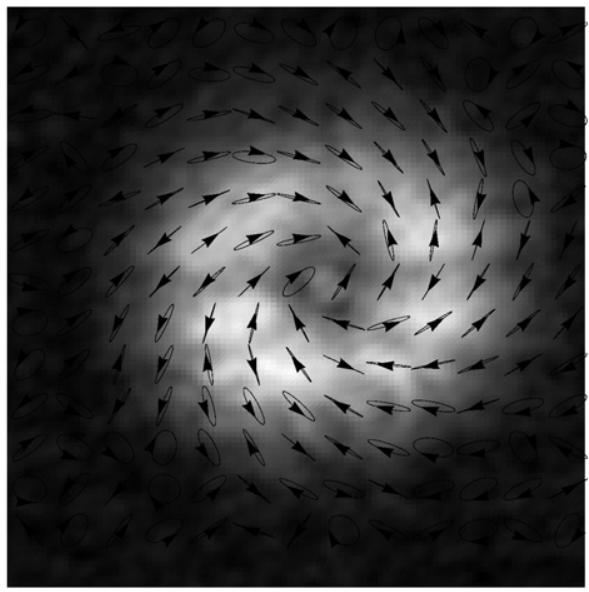

d

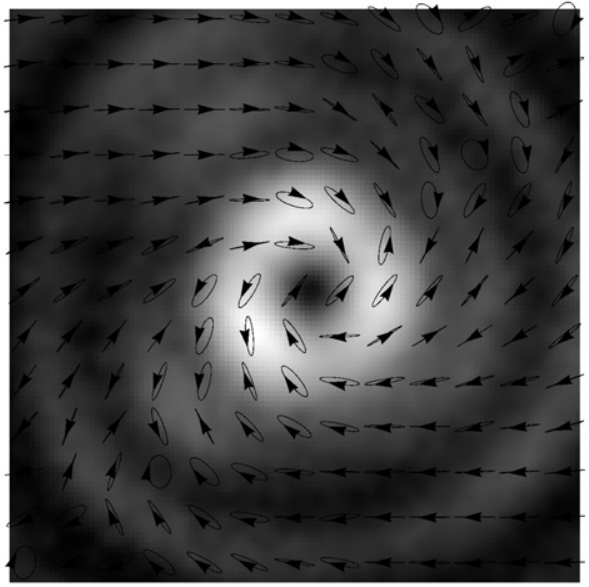

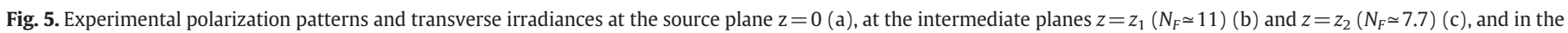
far field (d). 
a

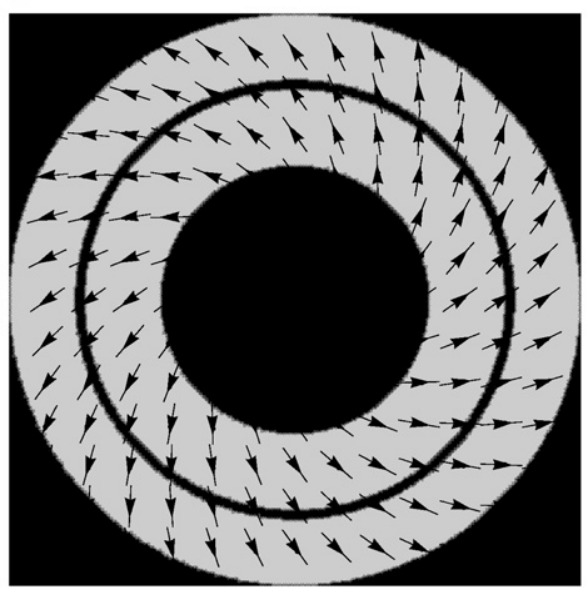

C

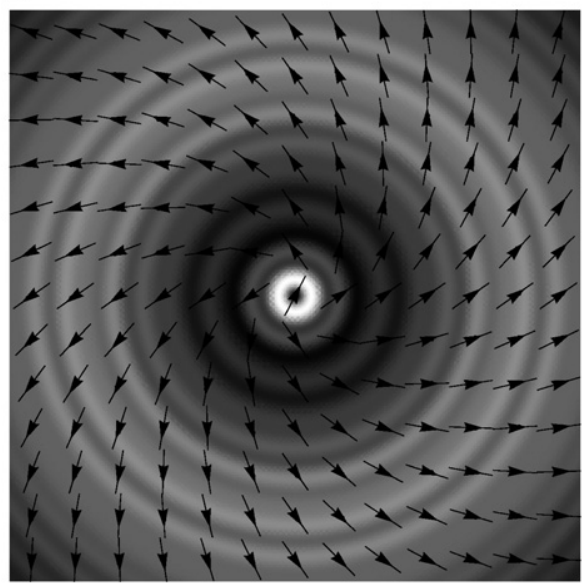

b

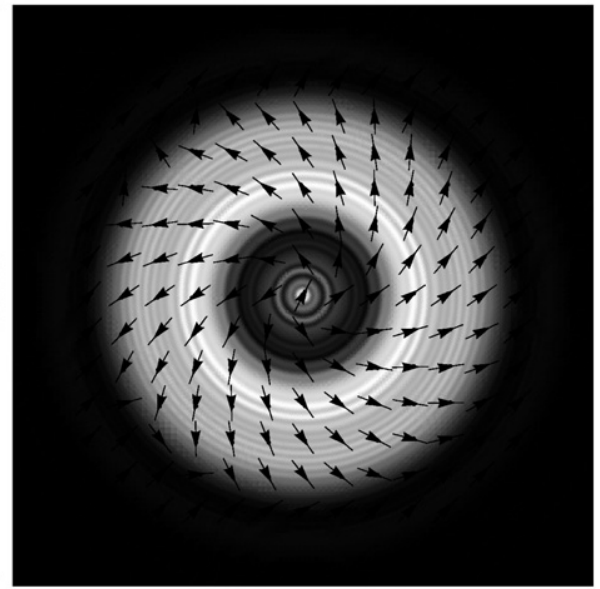

d

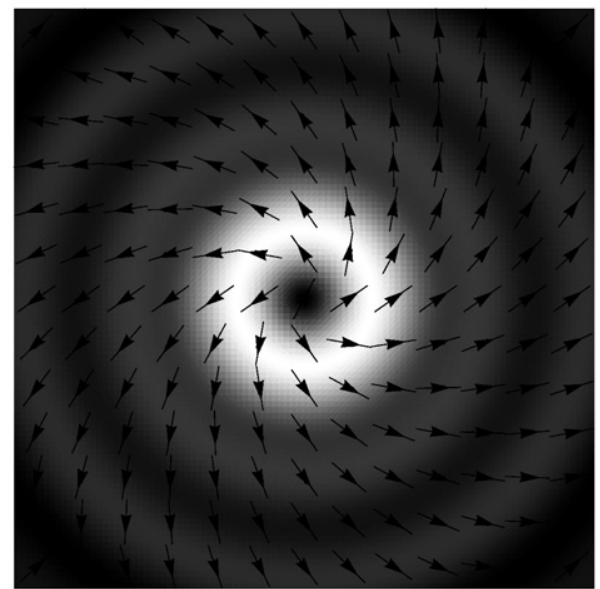

Fig. 6. Theoretical polarization patterns and transverse irradiances at the same planes than in Fig. 5.

From the polarization patterns shown in Fig. 5, it can be noticed that the synthesized beam presents a spiral-like, but slightly irregular distribution of polarization. For example, there are points of the beam profile where the polarization state is elliptic or where the azimuth angle is different from the expected one. It would be interesting to evaluate the quality or the uniformity of the polarization distribution of the synthesized beam by giving some parameters that characterize the beam, as far as its global features are concerned. A possible choice consists in measuring the spatial averages of the Stokes parameters across a beam section, and eventually represent them on the Poincaré sphere. In the case of spirally polarized beams, however, all the averaged values of the Stokes parameters, except $s_{0}$, are expected to vanish, and they cannot give any information about the distribution of the polarization across the beam profile. On the contrary, it seems more appropriate to use those global parameters that give the radial and azimuthal polarization contents across the transverse section of the beam $[20,31]$. Such parameters are denoted by $\tilde{\boldsymbol{\rho}_{r}}$ and $\tilde{\boldsymbol{\rho}_{\theta}}$, respectively, and are defined by means of the following expressions [20]:

$\tilde{\boldsymbol{\rho}}_{r}=\frac{1}{P_{T}} \int_{0}^{\infty} \int_{0}^{2 \pi} \rho r(r, \theta) I(r, \theta) r d r d \theta$

$\tilde{\boldsymbol{\rho}}_{\theta}=\frac{1}{P_{T}} \int_{0}^{\infty} \int_{0}^{2 \pi} \boldsymbol{\rho}_{r}(r, \theta) I(r, \theta) r d r d \theta$ where

$P_{T}=\int_{0}^{\infty} \int_{0}^{2 \pi} \rho_{r}(r, \theta) I(r, \theta) r d r d \theta$,

and $\boldsymbol{\rho}_{r}$ and $\boldsymbol{\rho}_{\theta}$ give the irradiance percentage of the radial and azimuthal component, respectively, of the field at each point across the beam profile.

It is useful to write these parameters in terms of the local Stokes parameters, $s_{i}(i=0,1,2,3)$, as [20]

$\tilde{\boldsymbol{\rho}}_{r}=\frac{1}{2}+\frac{1}{2 P_{T}} \int_{0}^{\infty} \int_{0}^{2 \pi}\left[\cos (2 \theta) s_{1}(r, \theta)+\sin (2 \theta) s_{2}(r, \theta)\right] r d r d \theta$

$\tilde{\boldsymbol{\rho}}_{\theta}=\frac{1}{2}-\frac{1}{2 P_{T}} \int_{0}^{\infty} \int_{0}^{2 \pi}\left[\cos (2 \theta) s_{1}(r, \theta)+\sin (2 \theta) s_{2}(r, \theta)\right] r d r d \theta$.

As can be easily seen from the above expressions, for fields written as in Eq. (7) the parameters defined in Eqs. (16)-(17) are independent of the field amplitude and turn out to be

$\tilde{\boldsymbol{\rho}}_{r}=\frac{1}{2}-\frac{1}{2} \cos (2 \alpha)$ 
Table 1

Experimental and theoretical values of parameters $\tilde{\boldsymbol{\rho}_{r}}, \tilde{\boldsymbol{\rho}_{\theta}}$ and $\tilde{\boldsymbol{\rho}_{c}}$ at different planes.

\begin{tabular}{llll}
\hline Plane & $\tilde{\boldsymbol{\rho}_{r}}$ & $\tilde{\boldsymbol{\rho}_{\theta}}$ & $\tilde{\boldsymbol{\rho}}_{c}$ \\
\hline$z=0$ & 0.33 & 0.67 & -0.03 \\
$z=z_{1}$ & 0.33 & 0.67 & -0.03 \\
$z=z_{2}$ & 0.36 & 0.64 & 0.02 \\
$z=R_{0}$ & 0.36 & 0.64 & -0.02 \\
Theoretical values & 0.33 & 0.67 & 0 \\
\hline
\end{tabular}

$\tilde{\boldsymbol{\rho}}_{\theta}=\frac{1}{2}+\frac{1}{2} \cos (2 \alpha)$.

Another global parameter can be useful for characterizing this kind of beams. Such parameter, namely $\tilde{\boldsymbol{\rho}}_{c}$, gives the circular polarization content across the beam profile, weighted with the values of the irradiance. In terms of the Stokes parameters, $\tilde{\boldsymbol{\rho}}_{c}$ is defined as [19]

$\tilde{\boldsymbol{\rho}}_{c}=\frac{1}{P_{T}} \int_{0}^{\infty} \int_{0}^{2 \pi} \boldsymbol{\rho}_{c}(r, \theta) s_{0}(r, \theta) r d r d \theta$

with

$\boldsymbol{\rho}_{c}(r, \theta)=\frac{s_{3}(r, \theta)}{s_{0}(r, \theta)}$.

It turns out that $\tilde{\boldsymbol{\rho}_{c}}=-1$ for pure left-handed circularly polarized light while $\tilde{\boldsymbol{\rho}}_{c}=+1$ for pure right-handed circularly polarized light. For fields with pure linear, even though non-uniform polarization, as is the case of spirally polarized fields, the value $\rho_{c}=0$ is expected.

For the generated beam, all these parameters have been calculated at the four transverse planes that we considered above: the source plane $(z=0)$, two intermediate planes $\left(z_{1}\right.$ and $\left.z_{2}\right)$ and the plane that corresponds to the far field $\left(z=R_{0} \mathrm{~mm}\right)$. Experimental values of these global parameters are shown and compared with the theoretical values in Table 1 . As can be seen in the table, the values of $\tilde{\rho_{r}}$ and $\tilde{\boldsymbol{\rho}_{\theta}}$ remain almost invariant along the four considered planes, meaning that the radial and the azimuthal polarization contents of the beam do not change appreciably upon free propagation. Furthermore, the value of $\tilde{\rho}_{c}$ shows that the content of circular polarization across the four planes is always less than (or equal to) $3 \%$.

The overall parameters studied in this section were already used for carrying out the global characterization of completely coherent spirally polarized fields [31]. In this section it has been shown that these parameters can be also useful for characterizing non-uniformly polarized partially coherent beams.

\section{Conclusions}

An experimental method for generating partially coherent nonuniformly polarized fields with propagation-invariant transverse polarization pattern has been presented. The method has been applied to the synthesis of a partially coherent beam with a spiral-like distribution of polarization. The synthesized beam has been characterized from two different points of view: by plotting the experimental polarization and irradiance patterns, evaluated from the measured local Stokes parameters at each point of the transverse section of the beam, and by means of some global parameters. It has been shown that the polarization patterns and the values of these global parameters do not change appreciably upon free propagation. Comparisons with the expected theoretical values in both cases have been given.

\section{Acknowledgments}

G. Piquero and V. Ramírez acknowledge the support from the Ministerio de Educación y Ciencia of Spain, project FIS2007-63396. M. Santarsiero wish to thank R. Martínez-Herrero, P. Mejías, J. Serna, G. Piquero and V. Ramírez for their kind hospitality during his stay at Universidad Complutense de Madrid.

\section{References}

[1] D.F.V. James, J. Opt. Soc. Am. A 11 (1994) 1641.

[2] G.P. Agrawal, E. Wolf, J. Opt. Soc. Am. A 17 (2000) 2019

[3] F. Gori, M. Santarsiero, R. Borghi, G. Piquero, Opt. Lett. 25 (2000) 1291.

[4] F. Gori, M. Santarsiero, G. Piquero, R. Borghi, A. Mondello, R. Simon, J. Opt. A: Pure Appl. Opt. 3 (2001) 1.

[5] F. Gori, J. Opt. Soc. A 18 (2001) 1612

[6] J. Tervo, J. Opt. Soc. Am. A 20 (2003) 1974.

[7] T. Shirai, Opt. Commun. 256 (2005) 197.

[8] O. Korotkova, E. Wolf, Opt. Commun. 246 (2005) 35.

[9] R. Martínez-Herrero, P.M. Mejías, Opt. Commun. 279 (2007) 20.

[10] E. Wolf, Opt. Lett. 32 (2007) 3400.

[11] X. Du, D. Zhao, Opt. Express 16 (2008) 16172

[12] F. Gori, Opt. Lett. 33 (2008) 2818.

[13] D.M. Zhao, E. Wolf, Opt. Commun. 281 (2008) 3067.

[14] Q. Zhan, Adv. Opt. Photon. 1 (2009) 1.

[15] R. Martínez-Herrero, P.M. Mejías, G. Piquero, Characterization of Partially Polarized Light Fields, Springer Series in Optical Sciences, 147, 2009.

[16] R. Borghi, M. Santarsiero, J. Opt. Soc. A 21 (2004) 2029.

[17] R. Borghi, M. Santarsiero, M.A. Alonso, J. Opt. Soc. A 22 (2005) 1420.

[18] V. Ramírez-Sánchez, G. Piquero, J. Opt. A: Pure and Appl. Opt. 10 (2008) 125004.

[19] R. Martínez-Herrero, P.M. Mejías, G. Piquero, Opt. Commun. 265 (2006) 6.

[20] R. Martínez-Herrero, P.M. Mejías, G. Piquero, V. Ramírez-Sánchez, Opt. Commun. 281 (2008) 1976.

[21] E. Wolf, Introduction to the Theory of Coherence and Polarization of Light, Cambridge University Press, 2007.

[22] M. Abramowitz, I. Stegun, Handbook of Mathematical Functions, Dover, 1972.

[23] P. de Santis, F. Gori, G. Guattari, C. Palma, Opt. Commun. 29 (1979) 256.

[24] M. Santarsiero, R. Borghi, V. Ramírez-Sánchez, J. Opt. Soc. Am. A 26 (2009) 1437.

[25] R. Martínez-Herrero, Nuovo Cimento 54 (1979) 205.

[26] E. Wolf, J. Opt. Soc. Am. 72 (1982) 343.

[27] M. Stalder, M. Schadt, Opt. Lett. 21 (1996) 1948.

[28] Q. Zhan, J.R. Leger, Opt. Express 10 (2002) 324.

[29] A.J. Campillo, J.E. Pearson, S.L. Shapiro, N.J. Terrel, Appl. Phys. Lett. 23 (1973) 85.

[30] M. Born, E. Wolf, Principles of Optics, 7th (expanded) ed, Cambridge U. Press, Cambridge, 1999.

[31] V. Ramírez-Sánchez, G. Piquero, M. Santarsiero, J. Opt. A: Pure Appl. Opt. 11 (2009) 085708 . 